\title{
A spectrotemporal analysis of DCN single unit responses to wideband noise in guinea pig
}

\author{
Patricia M. Backoff and Ben M. Clopton \\ Kresge Hearing Research Institute, Unwersty of Michigan, Ann Arbor, Michigan, U.S.A.
}

(Received 11 April 1990; accepted 24 November 1990)

\begin{abstract}
Spectrotemporal receptive fields (STRFs) were estimated for chopper and pauser units recorded in guinea pig dorsal cochlear nucleus (DCN). Sixteen wideband, periodic noise stimuli, represented as time-frequency surfaces of energy density, were crosscorrelated in time with the unit's corresponding period histograms to determine if specific energy patterns tended to precede spike occurrence. The STRFs obtained were unique to the DCN, as compared to the ventral cochlear nucleus (VCN) [Clopton and Backoff. 1991, Hear. Res. 52, 329-344] in their degree of temporal and spectral complexity. Certain unit response types, classified from their peristimulus-time histograms (PSTHs) to tonebursts, were associated with distinctive patterns in the STRFs. All STRFs had at least one region of elevated energy density (peak region) closely preceding spike occurrence, which may reflect a short-pathway, primary excitatory input (or inputs) to the neuron. In addition, some units displayed low-energy regions (troughs) with greater temporal precedences on their STRFs, particularly when higher stimulus intensities were used. This analysis approach appears to have potential for investigating functional neural connectivity and predicting responses to novel complex stimuli. although specific implementations of the technique impose limitations on the interpretation of results.
\end{abstract}

Spectrotemporal analysıs: Receptive field; Dorsal cochlear nucleus; Noise: Guinea pig

\section{Introduction}

In the past, tonal stimuli have been used almost exclusively to characterize the response properties of neurons in the auditory nerve and cochlear nucleus $(\mathrm{CN})$. Standard characterization approaches, based on measures of spike rate or just detectable changes in spike rate (Evans and Nelson, 1973; Young and Brownell, 1976; Young and Voigt, 1982), have demonstrated complex frequency response properties of DCN neurons, such as those unit types dominated by inhibitory frequency regions (Type IV). Other tone-based techniques have focused on temporal response properties which are unique to DCN neurons, such as the patterning of spike discharge for buildup, pauser and chopper-type units (Kiang et al., 1965a:

Correspondence to: Patricia M. Backoff, (Present address) Southern Illinois University School of Medicine, Department of Pharmacology, P.O. Box 19230. Springfield, IL 62794-9230. U.S.A. FAX: (2.17) 782.-0989.
Pfeiffer, 1966; Godfrey et al, 1975; Rhode and Smith, 1986). Measurements of phaselocking to tonal stimuli (Goldberg and Brownell. 1973; Johnson, 1980), and temporal coding for wideband stimuli related to partial synchrony, have been primarily studied in auditory nerve fibers (Kiang et al., 1965b; Ruggero, 1973; de Boer and de Jongh, 1978); however, few units in guinea pig DCN show strong synchronization.

Typically, temporal and frequency response properties have been studied independently in the classical tone-based approaches; yet for complex signal encoding at the level of the DCN, these properties may not be independent processes. In preliminary studies (Backoff and Clopton. 1989). distinctive temporal patterning of unit firing was seen in the unit period histograms (PHs) in response to periodic noise stimuli (Fig. 1). This patterning, indicating that most units responded with distinctive and replicable fluctuations in spike probability over a few hundred microseconds, was poorly described by measures of average spike 
rate. That is, patterning evoked by the noise stimulus often could be seen at levels which did not produce a significant change in the average rate from the baseline spontaneous rate.

The spectrotemporal analysis approach used in the present study, which has been described by Clopton and Backoff $(1989,1991)$, is an extension of the work of de Boer, Johannesma, Eggermont and their colleagues (reviewed by Eggermont et al., 1983). The underlying concept can be summarized as follows: neurons, driven by a sound stimulus having variations in its spectral energy over time, at any moment are assumed to respond in proportion to the appearance of a timefrequency stimulus pattern immediately preceding that moment. For a spike train, this response measure might be an estimate of a spike-rate function over repeated stimulus presentations. The stimulus energy immediately preceding a spike, when weighted for the probability of spike occurrence and represented in time and frequency coordinates, is termed that neuron's spectrotemporal receptive field (STRF). Eggermont and co-workers have obtained STRFs in the grassfrog auditory midbrain using tonepip stimuli (Eggermont et al., 1981; Epping and Eggermont, 1985), and a noise stimulus (Hermes et al., 1981), but only a cursory application of this approach has been made to the mammalian (cat VCN) auditory system (Eggermont et al., 1983).

The STRF approach provides an estimation of both temporal and frequency response properties, and provides information about the relationship between second-order stimulus properties and spike discharge. In conjunction with the Revcor function of de Boer (de Boer and de Jongh, 1978), aspects of both first- and second-order relationships with a noise stimulus are represented. Also, since this method utilizes a different class of stimuli (flat spectrum, periodic wideband noises), it may assess different aspects of neural processing than tonehurst stimuli. In this study, period histograms ( $\mathrm{PHs}$ ) were obtained for single units in the guinea pig $\mathrm{DCN}$ in response to a series of 16 periodic, digitally synthesized, wideband noises. Units were classified into one of the major response types based on their PSTH to tonebursts at the unit characteristic frequency (CF) (Godfrey et al., 1975; Rhode and Smith, 1986). This paper will focus on the two predominant response classes observed in the guinea pig DCN with this preparation: pauser-types, which represent approximately $50 \%$ of the units in the fusiform cell layer (FCL), and chopper-types which comprise over $40 \%$ of the classifiable units recorded in the superficial and deep layers (unpublished observations). For the analysis, the stimuli were transformed into time-frequency surfaces of energy density, and these surfaces crosscorrelated with the PHs (Clopton and Backoff, 1989; 1991). This produced a weighted stimulus surface, preceding the time of spike occurrence, corresponding to an estimate of the unit's STRF. Some of the unique patterns of receptive fields seen for pauser and chopper units will be described, and the implications for neural encoding discussed.

\section{Methods}

\section{Physiological preparation}

Single-unit responses were recorded from 16 adult pigmented guinea pigs ranging in weight from $220-400 \mathrm{~g}$. Anesthesia was induced with an intramuscular injection of ketamine hydrochloride (Vetalar, $100 \mathrm{mg} / \mathrm{kg}$ ) and xylazine (Rompun, 5 $\mathrm{mg} / \mathrm{kg}$ ), and maintained with regular supplements. To obtain a high degree of head stability, the skull was fixed to a rigid bar with a stainless steel bolt mounted on the dorsal cranium with dental acrylic. The pinna and a portion of the cxternal meatus were excised, and a Beycr DT-48 earphone, enclosed in an aluminum chamber acoustically damped with steel wool. was coupled directly to the external meatus to form a closed system. The posterior cranium was opened, and the cerebellum overlying the cochlear nucleus complex was aspirated. This allowed visual guidance of electrode placement on the surface of the DCN, including control of entry point and angle of penetration. The choice of entry point determined the tonotopic region to be sampled. The surgical defect was then filled with warm $2 \%$ agar in physiologic saline, to prevent dessication and reduce brain pulsations. The animal was placed on a DC-powered heating pad, and core body temperature was maintained at $37.5^{\circ} \mathrm{C}$ using feedback from a temperature probe. 


\section{Stimulı}

A set of 16 periodic wideband stimuli were digitally synthesized from their complex frequency spectrum using an inverse Fourier transform (Clopton and Backoff, 1991). Their waveforms were output at a rate of $50 \mathrm{kHz}$ over 8192 12-bit samples through a Modular Instruments M206 waveform register. Each stimulus had a period of $163.84 \mathrm{~ms}$. Their phase spectra were independently randomized. and the amplitude spectra had equal amplitudes for each Fourier coefficient between 299 and $12207 \mathrm{~Hz}$ with a $6.1 \mathrm{~Hz}$ resolution, but were zero outside this frequency band.

The noise stimuli were presented at an average spectral level of 0 to $30 \mathrm{~dB}$ rms re $20 \mu \mathrm{N} / \mathrm{m}^{2}$ (maximum possible output of $71.2 \mathrm{~dB} \mathrm{rms}$ at zero attenuation), or approximately $15 \mathrm{~dB}$ above the threshold for driving. The response characteristics of the coupled acoustic system were determined via acoustical calibration with a $B$ and $K$ half-inch microphone and calibrated probe tube. In later experiments. the noise stimuli were resynthesized and digitally compensated for nonuniform spectral amplitudes and phase distortion in the transduced stimuli Clopton and Backoff. 1991). A five-octave logarithmic tonal sweep was commonly used as a search stimulus, and a measure of spontaneous activity was taken for all units. In addition, responses to both low-to-high (upsweep) and high-to-low (downsweep) tonal sweeps, and iso-intensity/rate functions for $40 \mathrm{~ms}$ tonebursts at $C F$ were also collected for most units. CF was determined manually by adjusting tone-burst frequency and attenuation to estimate the frequency associated with the minimal threshold. and all units were classified on the basis of their PSTH responses to suprathreshold CF tonebursts. Although all major response types were encountered. pauser-type (pauser and pauser-buildup) and chopper-type units (sustaincd, or Chop-S, and irregular, or Chop-T) were preferentially selected for this study.

\section{Electrophysiological recording}

Glass micropipettes were pulled, filled with 2 $M$ potassium citrate, and beveled to give an impedance of 6-12 M $\Omega$. The electrode was advanced from outside a double-walled sound booth using a hydraulic microdrive until spikes from a single unit were isolated. Location of the electrode tip within a specific layer of the DCN was estimated from depth measurements and the site and angle of entry of the electrode relative to the surface. Spike waveforms were discriminated in amplitude with a level comparator set to obtain maximum superimposition of triggered spike waveforms on an oscilloscope. An 80286 processor with a Modular Instruments Incorporated laboratory interface was used for stimulus presentation and data collection, with all data analysis and graphical display accomplished off-line on the system used for stimulus synthesis.

\section{Data collection}

The times of spike occurrence were stored with an accuracy of $20 \mu$ s relative to the beginning of a stimulus period. In addition to on-line displays of period histograms. off-line analysis included PSTHs for tonebursts, PHs for noises and tone sweeps, interspike-interval histograms (ISIHs). average rate, and other analyses for all of the stimuli described. A PH for each of the 16 noise stimuli was usually collected based on 200 continuously presented stimulus periods. Examples of typical PHs are shown for two units in Fig. 1.

\section{Analysis}

The average and maximum discharge rates in silence and under driven conditions for each stimulus were measured for all units. Response latencies for the tonebursts at CF were also measured.

Details of our procedure for estimating the STRFs of individual units are given elsewhere (Clopton and Backoff. 1991). For the present study, the resolution of the analysis was increased. Previously, each noise was represented as a $128 \times$ 128 time-frequency matrix for the analysis, thereby limiting the frequency resolution of the STRF to $97.65 \mathrm{~Hz}$ and the time resolution to $1.28 \mathrm{~ms}$. For all STRFs reported here, however, each time function for a given reference frequency was calculated with a temporal resolution of $160 \mu$ s (1024 samples instead of 128 ). Each time function represented the variation in energy density as 'viewed' from its reference frequency, based on the real part of the Rihaczek distribution (Rihaczek. 1968). This form of stimulus representation was also used in the 
A

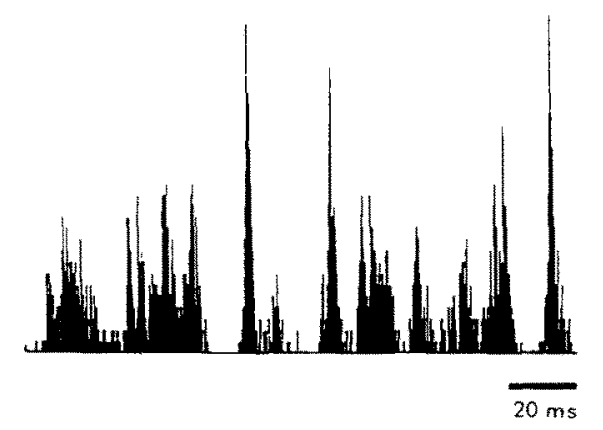

B

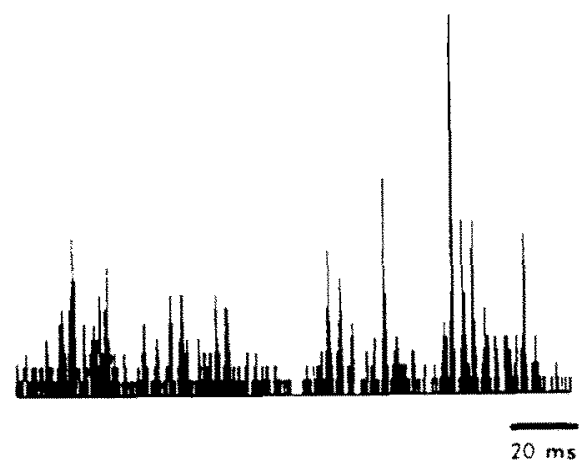

$20 \mathrm{~ms}$

Fig. 1. Period histograms for two DCN units, showing distinctive temporal patterning obtained in response to a periodic noise stimulus presented at a low intensity level (15dB above threshold). (A) A typical PH obtained for a pauser unit; the average discharge rate was 63.96 spikes/s (maximum rate $=$ 500.5 spikes/s). (B) $\mathrm{PH}$ response to the same stimulus obtained for a chopper unit; the average rate was $46.42 \mathrm{spikes} / \mathrm{s}$ (max, rate $=812.5$ spikes $/ \mathrm{s}$ ). The histograms were collected over 200 continuous presentations of the noise period (163.8 $\mathrm{ms})$, with a dwell time of $160 \mu \mathrm{s}$.

CoSTID of Johannesma (1972) and coworkers (Johannesma et al., 1981). To calculate the STRF, each time function for 128 ordered reference frequencies were crosscorrelated with the corresponding unit $\mathrm{PH}$. An average of the spectrotemporal energy-density pattern preceding a spike was obtained.

A value for any periodic stimulus waveform can be represented as the sum of the waveform's Fourier components at that time, that is, each one of its Fourier coefficients time-translated appropriately. If neighboring Fourier components are phase coherent, a higher than average energy value (peak) would occur, while components which are out of phase with each other would be associated with low energy regions (troughs). The correlation function across the noise series estimated the variations in energy which tended to precede spike occurrence for each reference frequency.

A

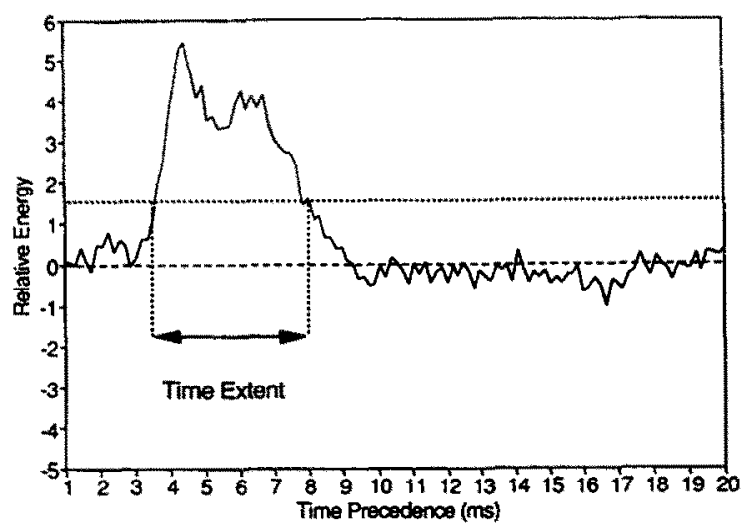

B

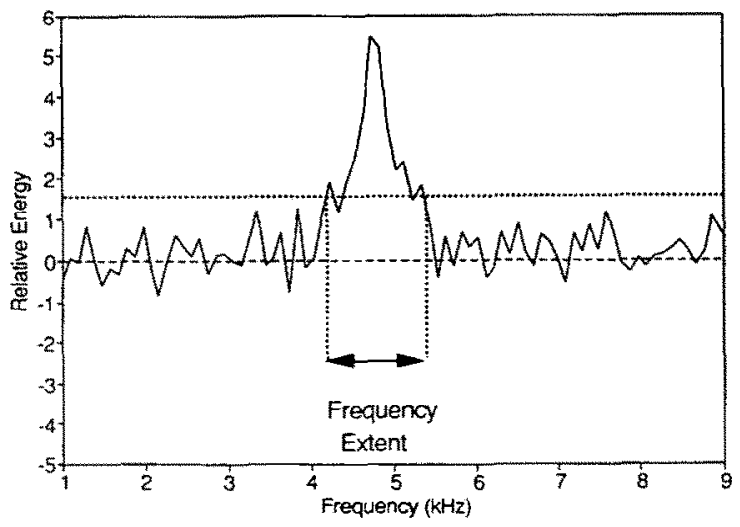

Fig. 2. The method used to calculate time and frequency extents of the major STRF feature areas are shown in $A$ and $B$, respectively. (A) A slice in time is taken through the STRF response surface at the reference frequency corresponding to the unit's best frequency (the frequency corresponding to the maximum peak, $B F_{\text {STRF }}$ ). Precedence time is shown on the $x$-axis, and relative peak energy in standard deviations (re: mean surface level of 0 ) shown on the $y$-axis. (B) A frequency slice taken through the precedence time associated with the maximum peak (tau), with frequency displayed along the $x$-axis, but the same relative energy units on the $y$-axis as in $A$. In both $A$ and $B$, measurements were taken between the two points on the curve intersected by the dashed 1.5 sigma line. 
When taken across all of the reference frequencies, the resulting spectrotemporal pattern described the unit's STRF.

The STRFs are displayed as two-dimensional contour plots in time and frequency. The horizontal axis represents time (tau) relative to the occurrence of a neural spike at tau $=0$. Positive values of tau correspond to time preceding the spike. Reference frequencies are displayed linearly along the vertical axis. The equal-energy contours are plotted in standard deviations above (for peaks, shown as stippled regions in the figures) and below (for troughs, as non-stippled areas) the mean STRF surface value. A standard minimum criterion level of 1.5 standard deviations from the mean surface value was chosen to show the greatest number of features possible without picking up obscuring background variation.

Measurements of the frequency and temporal extent of the STRF primary peak region (i.e. the feature region on the STRF containing the maximum power peak) were calculated for each unit using the method shown in Fig. 2. Fig. 2A shows a time slice through the STRF at the estimated best frequency for a pauser unit. The energy level of the mean background surface has been set to zero for reference, and the numbers above and below represent relative energy in standard deviations. The points at which the 1.5 criterion level intersects the unit's time reponse were used to determine the temporal extent. Similarly, the frequency extent of the primary peak area was determined using the same 1.5 sigma criterion, as illustrated in Fig. 2B. The frequency slice shown in this figure was taken through the STRF at a point in time which corresponded to the precedence value (tau) associated with the maximum peak on the STRF surface.

\section{Results}

This report summarizes a detailed examination of the STRFs for 52 single units (26 pauser-type. 26 chopper-type) isolated in the DCN. Half of the chopper units showed a sustained or regular chop pattern; the rest were irrcgular choppers (i.e. Chop-T). Units with a wide range of CFs (961$12.500 \mathrm{~Hz}$ ) were sampled. These units were chosen from a larger sample of several hundred in the $\mathrm{CN}$ from which we have successfully estimated STRFs.

STRFs could be obtained for all DCN units if they were driven by the noise set and had CFs within the noise bandlimits. The noise stimuli evoked distinctive patterns of spike activity in the period histograms for all driven units (e.g., Fig. 1). At the intensity levels used in this study, less than 3 percent of the units encountered were so strongly inhibited by the noise stimuli as to prevent calculation of a STRF. The average driven firing rate for the noise set (at $0-30 \mathrm{~dB}$ spectrum level) varied across units, from a low of 7.53 spikes/s to a maximum of 109.19 spikes/s. For two units (Chop-T unit in superficial DCN and a pauser in deep DCN), the noise reduced the average spontaneous firing rate by more than $20 \%$, although temporal patterning of the spike discharges was clearly evident in the $\mathrm{PH}$ records. An additional three pauser units also showed distinctly patterned PH responses to the noises without a significant change in firing rate from the spontaneous. All five of these units showed well-defined response regions on their STRFs.

Restricted regions of elevated energy density, or peak regions. on the receptive-field surfaces were observed for all driven DCN units. The relative energy levels associated with these peaks ranged from 2 to 9 sigma above the mean background surface level (1.6-5 dB above the mean) for this set of noise stimuli at low intensities. Units which showed strongly patterned responses in their PHs, with high spike counts over a small number of bins, had well-defined STRFs with the highest maximum peaks. The frequency associated with the highest peak on the STRF surface, which can be designated the STRF estimate of "best frequency' ( $\mathrm{BF}_{\mathrm{SIRF}}$ ), was compared to the unit CF measured with tonebursts. Fig. 3 shows the correlation between these two measurements for a group of DCN units, which included a small subpopulation of synchronized units (maximum synchronization index was 0.55 ). Thus, at these lower stimulus intensities, unit CF was closely related to $\mathrm{BF}_{\text {STRF }}$ obtained with wideband stimuli, for both synchronized and nonsynchronized DCN units.

The STRF patterns seen for units in DCN were quite distinctive, with greater temporal complexity than the patterns typically seen for AVCN 
primarylike units (Clopton and Backoff, 1991). All DCN units showed at least one primary peak region, and many units showed additional peak, and sometimes trough, regions as well (Figs. 4, 5, 6; stippled regions represent peaks, troughs are denoted by clear contours). Generally, these peaks and troughs overlapped the same frequency range, with trough areas having greater time-precedence values than the shorter precedence peak regions. While quantitative details of the pattern features appeared to be unique to individual units, some general pattern features were seen within certain neural response classes. These similarities were especially evident among two particular classes of units, pauser-types and chopper-types. These units not only show temporal complexity in their responses to simple tonal stimuli, but also in their STRFs for wideband stimuli. In Fig. 4, STRF patterns typical of chopper units in the superficial and deep DCN, respectively, are shown in $A$ and $C$, while $B$ and $D$ show patterns typical of pausers recorded in the FCL. Typical STRFs for pausers showed a single primary peak region with a long

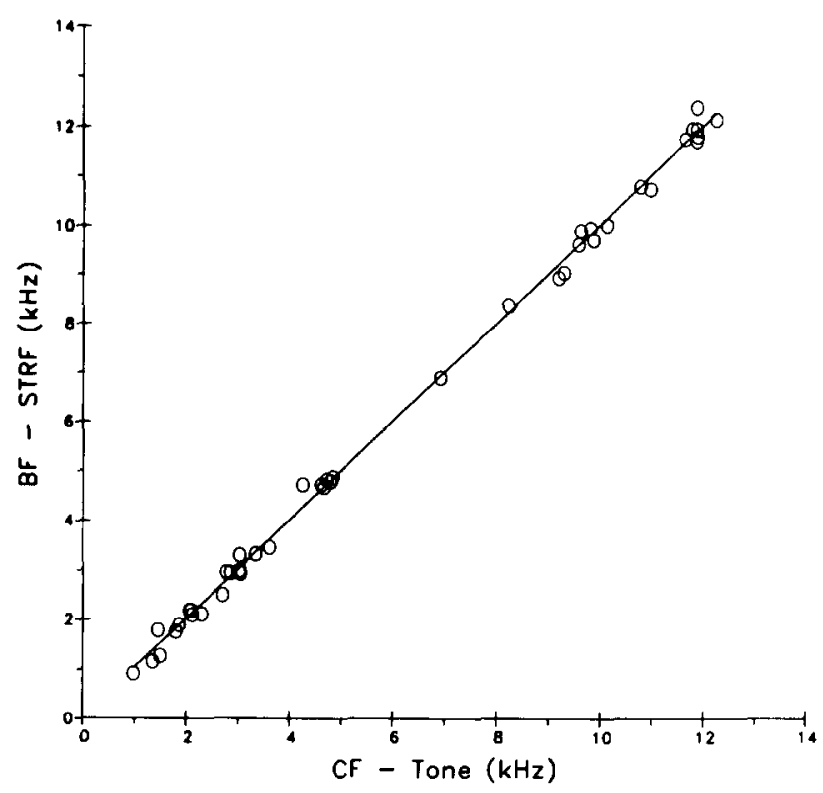

Fig. 3. Data from 42 units recorded in DCN (open circles), showing the relationship between the STRF estimate of unit best frequency and the CF determined manually with tonebursts. The linear regression line through the data points had a correlation coefficient of 0.9989 , revealing a direct correlation between these two measures. temporal extent ( $>2.4 \mathrm{~ms}$ ), while choppers tended to show a banded pattern of multiple peak regions or combinations of peaks and troughs. Twentythree of the 26 pausers $(88.5 \%)$ exhibited the typical long duration pattern, and 22 of the $26(85 \%)$ choppers exhibited banded patterns. All of the remaining chopper neurons and one pauser exhibited a single primary peak region with a relatively short temporal extent $(<2.4 \mathrm{~ms})$; the other two pausers showed banded patterns.

At the lower stimulus intensities which were used, chopper units showed a variety of banded patterns. The most common pattern consisted of two or more temporally separated peak regions, similar in their frequency and temporal extents. The second most common pattern was an interleaving of peak and trough bands. The number of bands seen in the STRF was not related to the number of 'chops' observed in the unit's PSTH response to tonebursts, nor did a particular banding pattern appear to be related to the type of chopper unit (either irregular or sustained), although a larger population sampling is needed. At least for some units, the interval between major peak bands, measured at best frequency, did correspond to the 'chop interval' of the unit's toneburst response (Fig. 5A). Other units did not show a clear correspondence between the STRF band interval and the PSTH chop interval, at least for the stimulus intensities used (Fig. 5B). In over half of the banded STRFs, and most STRFs in general, the primary excitatory peak region occurred with the shortest precedence (tau) relative to the time of neural firing, presumably representing the shortest input pathway to the neuron. For other neurons, particularly at higher intensity levels, the secondary peak region (having a lower maximum power peak than the primary region) appeared at a shorter time-precedence than the primary peak arca.

The major features of the STRF patterns were quite robust for individual units. Replicable STRFs containing all of the major response features could be obtained using only half of the stimulus set, or by halving the number of presentations for each of the noise stimuli in the original set (Clopton and Backoff, 1991).

Table I shows summary data for frequency extent, temporal extent, and tau (precedence time 
for the maximum power peak) for groups of DCN pauser and chopper units. Pauser units showed the greatest time-precedence values, frequently with maximum peaks occurring $10-11 \mathrm{~ms}$ before the spike. The range of temporal extents of the primary peak region showed some overlap between the two classes, but the differences were statistically significant (at $P=0.005$ level. Student's $t$-test), with longer temporal extents for pausers than for the chopper units. Comparison of frequency extents for the primary peak regions again showed significant differences between these two unit classes. Pausers had more restricted STRF frequency extents than choppers, often as narrow as a few hundred Hertz. The frequency extents measured for these response areas may reflect the low stimulus intensities used.
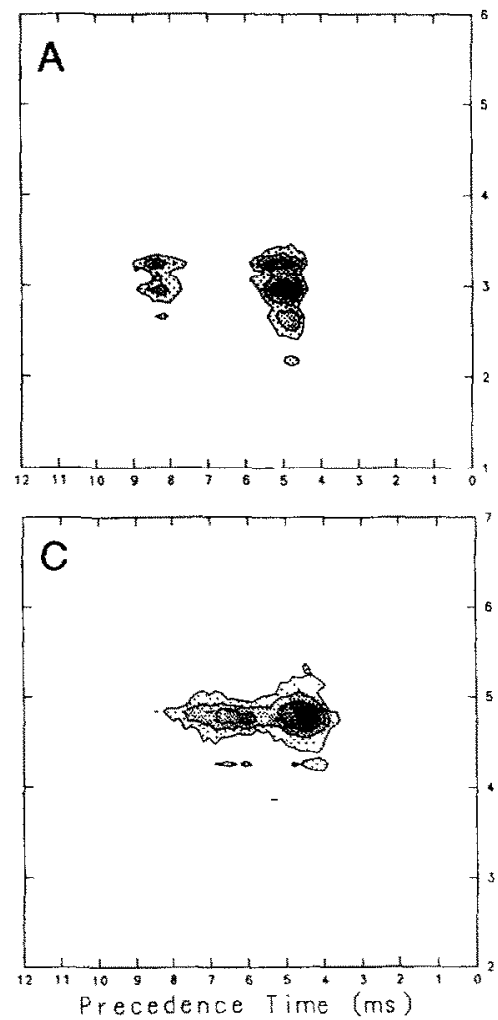

For a few units, STRFs were obtained at one or two additional intensity levels, moderate levels corresponding to $40-55 \mathrm{~dB}$ spectrum level. While the number of units sampled was small, every unit showed changes in frequency extent and/or pattern associated with the increase in stimulus intensity. Some units showed a reduction in frequency extent while others showed broader response areas. Changes in the STRF pattern, however, were seen for nearly every unit tested with intensity increases of the noise stimuli as small as 5 or $10 \mathrm{~dB}$. Fig. 6 shows the two types of changes which were commonly observed. First, there was often a change in the overall STRF pattern, seen either as changes in pattern-type (i.e., from a single long duration peak shown in $6 \mathrm{~A}$, to the banded pattern shown in $6 \mathrm{~B}$ ), or the addition of peaks to banded
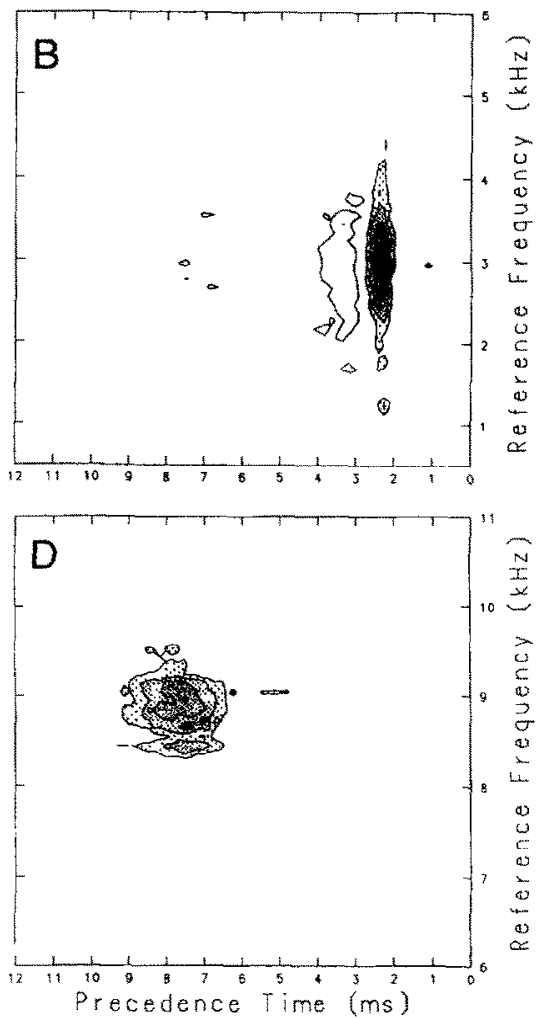

Fig. 4. Examples of typical STRF patterns for DCN chopper-type (upper panels A, B) and pauser-type units (lower panels C, D). (A) Banded pattern of two peak regions, for a chopper in superficial DCN (CF $=2783 \mathrm{~Hz})$. (B) Banded pattern of peak and trough regions, for a chopper in deep DCN $(\mathrm{CF}=3026 \mathrm{~Hz}$ ). (C) Long duration peak region, for an $\mathrm{FCL}$ pauser unit $(\mathrm{CF}=4780 \mathrm{~Hz})$. (D) Long duration peak for another pauser recorded in the FCL $(C F=9219 \mathrm{~Hz}$ ). For all STRF figures (Figs. 4-6). peak areas are displayed on a stippled gray scale, with the darkest region corresponding to the highest peak; the outlined unstippled tegions indicate troughs. 

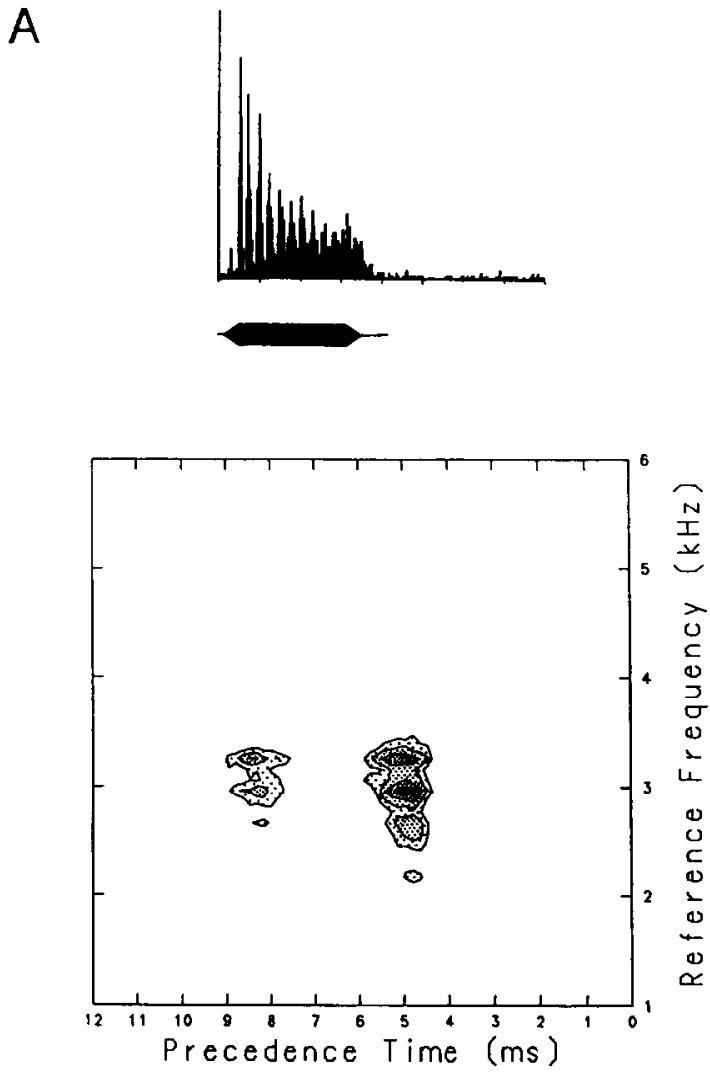
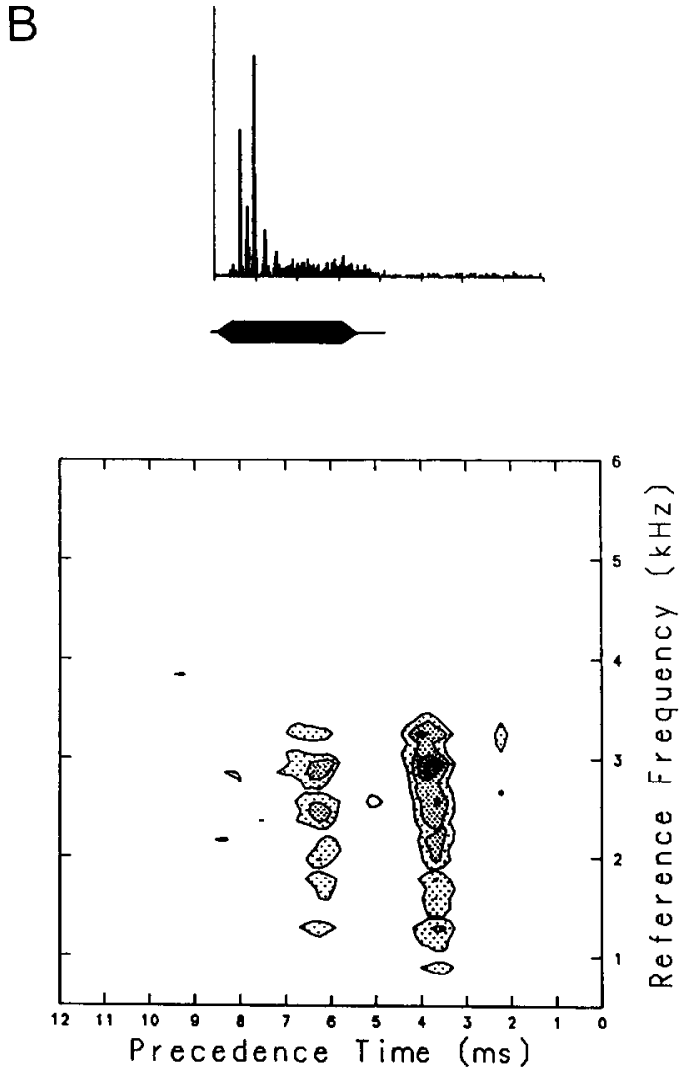

Fig. 5. Relationship between PSTH 'chop interval' and the interval between major STRF peak bands for two DCN choppers of similar CF. The PSTH for a $40 \mathrm{~ms}$ toneburst at CF is shown at the top. and an expanded view of the STRF surface is shown at the bottom of each panel. (A) Sustained chopper: the STRF band interval equals the PSTH chop interval (3.2 ms). (B) Irregular chopper: the STRF band interval was slightly longer than the PSTH chop interval ( $2.76 \mathrm{~ms}$ vs $2.3 \mathrm{~ms}$ ). Points used to determine band intervals were taken from time slices at $\mathrm{BF}_{\mathrm{STRF}}$, at the tau values corresponding to the highest peak in each band.

patterns for choppers. The second typical pattern change (also shown in Fig. 6B) was the appearance of trough regions at the higher intensities.

\section{Discussion}

The spectrotemporal analysis described in this study produced distinctive and replicable STRFs for neurons in the guinea pig DCN. This proved possible for almost all of the units encountered, given that they responded to frequencies within the bandlimits of the noise, except for a few neurons which were strongly inhibited by the noise stimuli. The presence of distinct, temporally patterned spike discharges in the unit PHs was a better indicator of a unit's responsiveness to the noise stimuli than changes in avcrage firing rate from the spontaneous. The results indicate that spikes evoked in these neurons by the noise stimuli were associated with specific patterns of energy fluctuation. The patterns consisted of brief increments or decrements in energy restricted in frequency content shortly preceding the spikes, generally by intervals less than $10 \mathrm{~ms}$. The frequency maxima of the STRF surfaces were found to correspond to the unit CF estimated from toneburst responses. While the individual pattern features showed a range of temporal extents, the duration of the major peak region was usually between 1-4 ms.

Significant differences in response pattern, temporal and frequency extents were seen between two major physiological unit classes, pausers and 
choppers. Although general pattern categories appear to be related to functional types characterized with tonebursts, individual patterns differed enough in their feature details to distinguish between different units within the same response class. Also, based on limited observations, it appears that increasing stimulus intensity modifies both peak and trough features in a pattern. often with the expansion of existing trough regions or the addition of new ones.

For these data, there was a trend towards narrower peak receptive field areas for the pauser units, at least for the low stimulus intensities used. which is consistent with the narrow excitatory regions seen with toneburst stimulation for these neurons (Young and Voigt, 1982; Rhode and Smith, 1986; Young et al.. 1988). Small trough regions, centered near the unit's $\mathrm{BF}_{\mathrm{STRF}}$, have been observed in the STRFs of many pauser types. particularly at higher stimulus intensities. Many of the pauser-buildups recorded within the boundaries of the FCL probably represent fusiform cells: most of these units exhibited the characteristic non-monotonic rate-intensity functions for tonebursts, showed evidence of large inhibitory frequency regions. and were well-driven by the noise stimuli. Previous intracellular marking stud-

\section{TABLE I}

SUMMARY DATA FOR GROUPS OF DCN PAUSER AND CHOPPER UNITS

\begin{tabular}{|c|c|c|}
\hline Primary Peak & Pausers & Choppers \\
\hline \multicolumn{3}{|c|}{ Precedence (tau) } \\
\hline Range & $4.065-11.634 \mathrm{~s}$ & $1.960-7.216 \mathrm{~ms}$ \\
\hline Mean & $6.619 \mathrm{~ms}$ & $5.011 \mathrm{~ms}$ \\
\hline$\sigma$ & $1.687 \mathrm{~ms}$ & $1.320 \mathrm{~ms}$ \\
\hline $\mathrm{n}$ & 26 & 25 \\
\hline \multicolumn{3}{|c|}{ Temporal Extent $*$} \\
\hline Range & $0.952-5.758 \mathrm{~ms}$ & $0.711-2.522 \mathrm{~ms}$ \\
\hline Mean & $3.084 \mathrm{~ms}$ & $1.420 \mathrm{~ms}$ \\
\hline$\sigma$ & $1.337 \mathrm{~ms}$ & $0.415 \mathrm{~ms}$ \\
\hline $\mathrm{n}$ & 23 & 23 \\
\hline \multicolumn{3}{|c|}{ Frequency Extent * } \\
\hline Range & $195-1367 \mathrm{~Hz}$ & $675-2930 \mathrm{~Hz}$ \\
\hline Mean & $786 \mathrm{~Hz}$ & $1445 \mathrm{~Hz}$ \\
\hline$\sigma$ & $368 \mathrm{~Hz}$ & $541 \mathrm{~Hz}$ \\
\hline $\mathrm{n}$ & 22 & 22 \\
\hline
\end{tabular}
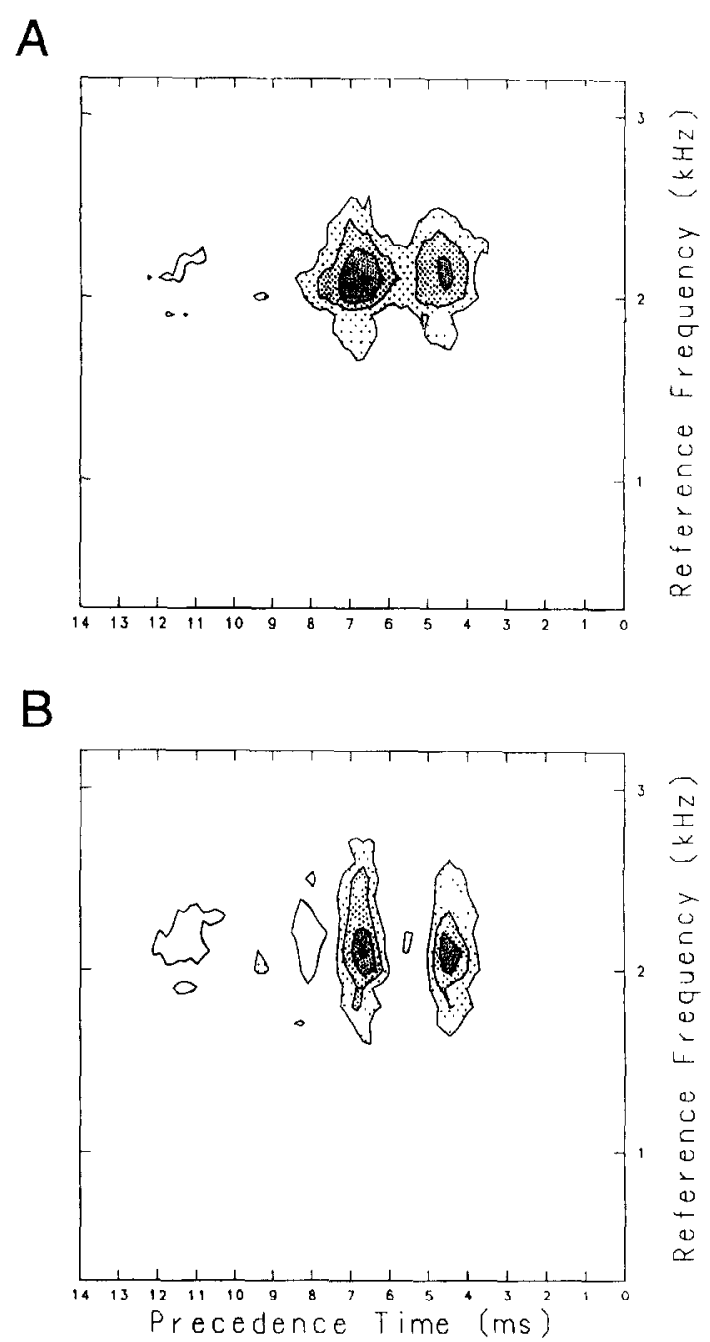

Fig. 6. The effect of a small change in stımulus intensity on the STRF pattern shown for an FCL pauser unit $(\mathrm{CF}=2097 \mathrm{~Hz})$. (A) At the lower intensity presentation level (36.2 dB spectrum level), the STRF shows the long duration response area typical of pauser units, but with a narrow trough region. (B) When stimulus intensity is increased by $10 \mathrm{~dB}$ (to $46.2 \mathrm{~dB}$ spectrum level), the STRF changes to a banded pattern of interleaving peaks and troughs.

ies (Rhode and Smith, 1986) have identified pauser-buildup type units as fusiforms; other studies have also associated Type IV response maps with this cell type. At first, one might be surprised that none of the FCL pauser-buildup STRFs show dominant trough regions, comparable to the large inhibitory regions of Type IV units for tonal stimuli; instead, the STRFs of these pauser units 
primarily show a long duration peak region. This is, however, entirely consistent with the strongly driven response of Type IVs for wideband noise stimuli (Young and Brownell, 1976) and with the suggested circuitry and neuronal interactions of Type II-Type IV neurons, which have been described by Voigt and Young (1980). In their work, crosscorrelation of simultaneously recorded spike trains from pairs of Type II/III-Type IV units has provided evidence of inhibitory interactions of Type II neurons on Type IV neurons for tonal stimulation. Under noise stimulation, however, one might expect a release of the inhibitory effect of Type II neurons, since these units are only weakly driven by noise whereas the Type IV units are vigorously driven by noise. Thus, for the low to moderate level noise stimuli used in the present study, one would expect the STRF of a Type IV unit to exhibit a major peak region centered at the CF (representing a primarily excitatory response), which indeed agrees with the current observations.

It is probably not valid to compare STRF-derived measurements of frequency extent with tonally derived response areas. First, the 1.5 sigma measurement criterion may not represent the optimal measurement level. It was selected because it represented the minimum level across all units which would detect the greatest number of features without picking up excessive background variation. An obvious alternative would be to set the minimum contours at $3 \mathrm{~dB}$ down from the STRF maximum power peak, which would be comparable to the estimation of bandwidth for a linear filter. However, due to the variability in the mean surface level and maximum peak height across units, the use of the 1.5 sigma criterion is preferable. Indeed for the majority of units, use of a $-3 \mathrm{~dB}$ criterion would have obscured many of the individual features on the STRF. When both measurement criteria could be used, similar values were found for frequency extent (usually within $200 \mathrm{~Hz}$ ) with each method, with no obvious trends. It should be noted, however, that the CFs of these units ranged between $1-5 \mathrm{kHz}$; it is always possible that higher $\mathrm{CF}$ units might show more consistent discrepancies between frequency extent estimation with these two methods, although we judge this unlikely. Using the 1.5 sigma measurement criterion, no direct relationship was seen between unit CF and frequency extent for the low intensity stimuli. The narrowest response areas were observed for high CF pauser-buildups, and also for low CF units which exhibited low-to-moderate phaselocking.

Second, these STRFs were obtained using only one to three intensity levels, whereas tonal response maps are obtained over a wide range of intensities. To facilitate any comparison, additional surfaces would need to be obtained at a number of intensities. Examination of STRFs over a greater intensity range would best describe changes in frequency extent which accompany an increase in stimulus intensity for this set of complex stimuli.

Finally, the basic differences in methodology and the stimuli used to obtain these two estimates of response area do not readily allow for direct comparisons between them. Tonal response maps focus on changes in firing rate as a function of frequency and intensity; for STRFs, temporallypatterned spike discharges are the best indication of a unit's responsiveness to the periodic noise stimuli, independent of changes in average firing rate. There is no correlate of temporal extent in tonal response maps, which appears to be a significant feature of STRF response areas. Also, differences in the two types of stimuli used, series of tonebursts presented as individual frequencies versus a series of multi-component stimuli, also limits comparisons of response areas estimated by these two methods.

Some complex temporal features, such as banding in the STRFs of choppers, reflect temporal response properties which are similar to those observed with toneburst stimuli. This was especially true for those choppers having banding intervals in the STRF which equaled the chop interval in the unit's PSTH for tonebursts. Other STRF patterns, such as the long-duration peak areas of pausers, are more distinctive, and may reflect the activation of mechanisms for this class of stimuli which differ from those for tonal stimulation.

A full interpretation of these results depends on physiological, anatomical, and mathematical considerations. Many have been addressed in the work of de Boer, Johannesma, Eggermont and others (Eggermont et al., 1983), as well as discussed in Clopton and Backoff (1991). At least three qualifi- 
cations bear on the implementation of the STRF as used in this study. First, although this stimulus representation is not unique and alternative energy distributions exist (Cohen. 1989), it was chosen because of previous work (Eggermont et al., 1983: Backoff and Clopton, 1989; Clopton and Backoff, 1989) and its favorable properties for implementation. Eggermont and Smith (1900) have examined the Wigner distribution, and we have made a preliminary evaluation of the Choi-Williams distribution (Choi and Williams, 1989) for analyzing data as presented here. These alternative time-frequency representations have theoretical advantages over the Rihaczek distribution, but their implementations can involve significant increases in computational time. Second, timefrequency patterns obtained with reverse-correlation may be biased by serial dependencies in neuronal firing patterns (Eggermont et al.. 1983). This was of particular concern in the case of units with banded STRFs; however, the contribution of multiple-spiking could be evaluated by examining the autocorrelation functions. Finally, STRFs portray second-order characteristics of the relationship between stimulus and response (Eggermont et al., 1983). Thus, if a neuron is sensitive to stimulus features of higher order. these must be estimated separately, for example, as conditional relationships on the STRF surface.

The interpretation of the peak and trough regions in these STRFs also requires further study. It is likely that the major peak area in the STRF of primarylike units, the only feature observed for them (Clopton and Backoff, 1991), corresponds to short-latency excitation expected for those neurons. For the DCN units in this study, particularly chopper units with banded STRFs, the presence of other peak areas at different precedence times might arise due to separate excitatory inputs to the neuron driven through pathways with a greater number of synapses. On the otherhand, they may reflect intrinsic membrane properties, such as a tendency of the neuron to generate multiple spikes at favored intervals (Kim et al., 1990). An intrinsic property of this nature would enhance responses to two or more energy peaks spaced at the favored interval. We have observed banded patterns in STRFs both in the absence of multiple-spiking and also for units which showed periodicities in their firing on the autocorrelation functions. Other explanations are possible, but these two provide contrasting mechanisms which are subject to experimental investigation.

Similar questions can be asked about the trough regions. An obvious working hypothesis is that they are associated with inhibitory inputs to the neuron, but a number of properties must be examined to support or refute this supposition. For example. one could examine whether application of pharmacologic agents known to block the action of putative inhibitory neurotransmitters in the DCN (Caspary et al., 1985, 1987) causes a change in the number, temporal or frequency extents of trough features on the STRF. It is also somewhat puzzling that trough areas are not observed lateral to the peak areas, as might be expected on the basis of the standard response maps obtained with tones (Evans and Nelson. 1973; Young and Brownell, 1976: Young and Voigt, 1982); instead, troughs usually overlap the same general frequency region as the peak areas (Clopton and Backoff, 1991). Again, this may in part be related to the low stimulus intensities used, suggesting an examination of STRFs over a wide intensity range. The lack of lateral trough regions in the STRF, however, may reflect basic differences in the stimuli used, one having distinct spectral contours (tones) and the other not (flatspectrum noise). Other possibilities, of course, include differences in the anesthetic agents used (here, ketamine/xylazine versus more commonly used barbiturates) or species differences (guinea pig versus cat), however studies could be easily designed to address these issues.

The resolution of such issues will determine the utility of this approach to understanding the physiology of the DCN. If the observed peak regions correspond to excitation and the trough regions to supression or inhibition, then STRFs provide information not available from unit responses to tonebursts, which is in agreement with previous arguments for the utility of this approach (Eggermont et al., 1981, 1983). For example, the effects of excitatory and inhibitory inputs within a common frequency range at the same stimulus intensity level could not be observed using tones where inhibition has commonly been identified as a decrement in spike rate from a spontaneous baseline. 
Also, if these temporally segregated excitatory and inhibitory regions on the STRF represent temporally (and spatially) separate input pathways to the neuron, and its unique synaptic and membrane properties, then basic questions of neural connectivity can be posed. The specific utility of the STRF technique in multi-unit recording is addressed in another study (Kipke et al., 1991). A functional measure of convergent inputs to a neuron as reflected in the STRF, in conjunction with established procedures and careful interpretation, can thus provide a basis for the analysis of neuronal networks in the auditory system and a more realistic description of how biologically important sounds are processed.

\section{Acknowledgements}

This work was supported by NIH grant NS05785 and equipment grant BNS8609850 from the NSF. This study comprises part of a doctoral dissertation, submitted in partial fulfillment of the degree requirements for the Doctor of Philosophy in the Horace H. Rackham School of Graduate Studies in The University of Michigan (dissertation microfilm to be submitted to the Library of Congress and University of Michigan Graduate Library).

\section{References}

Backoff, P.M. and Clopton, B.M. (1989) A time-frequency surface analysis of DCN and VCN unit responses to wideband noise. Abstr. Assoc. Res. Otolaryngol. 57.

de Boer, E. and de Jongh, H.R. (1978) On cochlear encoding: potentialities and limitations of the reverse correlation technique. J. Acous. Soc. Am. 63, 115-135.

Caspary, D.M. Rybak, L.P. and Faingold, C.L. (1985) The effects of inhibitory and excitatory amino acid neurotransmitters on the response properties of brainstem auditory neurons. In: D. Drescher (Ed.), Auditory Biochemistry. Charles C Thomas, Springfield, IL. 198-226.

Caspary, D.M., Pazara, K.E., Kössl, M. and Faingold, C.L. (1987) Strychnine alters the fusiform cell output from the dorsal cochlear nucleus. Brain Res. 417, 273-282.

Choi, H.-I., and Williams, W.J. (1989) Improved time-frequency representation of multicomponent signals using exponential kernels. IEEE Trans. Acoust. Speech Signal Proc. 37, 862871.

Clopton, B.M. and Backoff. P.M. (1989) Estimation of timefrequency energy patterns which stimulate neurons of the auditory system. Abstr. Assoc. Res. Otolaryngol. 56.
Clopton, B.M. and Backoff, P.M. (1991) Neural responses to spectrotemporal patterns in complex sounds. Hear. Res. 52, 329-344.

Cohen, L. (1989) Time-frequency distributions. Trans. IEEE Proc. 77, 941-981.

Eggermont, J.J., Aertsen, A.M.H.J., Hermes, D.J. and Johannesma, P.I.M. (1981) Spectro-temporal characterization of auditory neurons: redundant or necessary? Hear. Res. 5, 109-121.

Eggermont, J.J., Johannesma, P.I.M. and Aertsen, A.M.H.J. (1983) Reverse-correlation methods in auditory research. Quart. Rev. Biophys. 16, 341-414.

Eggermont, J.J. and Smith, G.M. (1990) Characterizing auditory neurons using the Wigner and Rihacek distributions: a comparison. J. Acous. Soc. Am. 87, 246-259.

Epping, W.J.M. and Eggermont, J.J. (1985) Relation of binaural interaction and spectro-temporal characteristics in the auditory midbrain of the grassfrog. Hear. Res. 19, 15-28.

Evans, E.F. and Nelson, P.G. (1973) The responses of single neurones in the cochlear nucleus of the cat as a function of their location and the anaesthetic state. Exptl. Brain Res. $17,428-442$.

Godfrey, D.A., Kiang, N.Y.S. and Norris, B.E. (1975) Single unit activity in the dorsal cochlear nucleus of the cat. $\mathrm{J}$. Comp. Neurol. 162, 269-284.

Goldberg, J.M. and Brownell, W.E. (1973) Discharge characteristics of neurons in the anteroventral and dorsal cochlear nuclei of cat. Brain Res. 64, 35-54.

Hermes, D.J., Aertsen, A.M.H.J., Johannesma, P.I.M. and Eggermont, J.J. (1981) Spectro-temporal characteristics of single units in the auditory midbrain of the lightly anaesthetised grass frog (Rana temporaria L.) investigated with noise stimuli. Hear. Res. 5, 147-178.

Johannesma, P.I.M. (1972) The pre-response stimulus ensemble of neurons in the cochlear nucleus. In: B.L. Cardozo (Ed.), Proc. IPO Symposium on Hearing Theory. IPO: Eindhoven, 58-69.

Johannesma, P., Aersten, A.D., Cranen, B. and van Erning, L. (1981) The phonochrome: a coherent spectro-temporal representation of sound. Hear. Res. 5, 123-145.

Johnson, D.H. (1980) The relationship between spike rate and synchrony in responses of auditory-nerve fibers to single tones. J. Acoust. Soc. Am. 68, 1115-1122.

Kiang, N.Y.S., Pfeiffer, R.R., Warr, W.B. and Backus, A.S.N. (1965a) Stimulus coding in the cochlear nucleus. Ann. Otol. Rhinol. Laryngol. 74, 463-485.

Kiang, N.Y.S., Watanabe, T., Thomas, E.C. and Clark, L.F. (1965b) Discharge patterns of single fibers in the cat's auditory nerve. MIT Press, Cambridge.

Kim, D.O., Sirianni, J.G. and Chang, S.O. (1990) Responses of DCN-PVCN neurons and auditory nerve fibers in unanesthetized decerebrate cats to AM and pure tones: Analysis with autocorrelation/power-spectrum. Hear. Res. 45, 95-113.

Kipke, D.R., Clopton, B.M. and Anderson, D.J. (1991) Common-stimulus driving and connectivity in groups of neurons in the dorsal cochlear nucleus. Hear. Res. (submitted). Pfeiffer, R.R. (1966) Classification of response patterns of 
spike discharges for units in the cochlear nucleus: tone-burst stimulation. Exptl. Brain Res. 1, 220-235.

Rhode, W.S. and Smith, P.H. (1986) Physiological studies on neurons in the dorsal cochlear nucleus of the cat. J. Neurophysiol. 56, 287-307.

Rihaczek, A.W. (1968) Signal energy distribution in time and frequency. IEEE Trans. IT 14, 369-374.

Ruggero, M.A. (1973) Response to noise of auditory nerve fibers in the squirrel monkey. J. Neurophysiol. 36, 569-587.

Voigt, H.F. and Young. E.D. (1980) Evidence of inhibitory interactions between neurons in the dorsal cochlear nucleus. J. Neurophysiol. 44, 7696.
Young, E.D. and Brownell, W.E. (1976) Responses to tones and noise of single cells in dorsal cochlear nucleus of unanesthetized cats. J. Neurophysiol. 39, 282-300

Young, E.D., Shofner, W.P., White, J.A., Robert, J. and Voigt, H.F. (1988) Response properties of cochlear nucleus neurons in relationship to physiological mechanisms. In: G.M. Edelman, W.E. Gall and W.M. Cowan (Eds.), Auditory Function: Neurobiological Bases of Hearing. John Wiley and Sons, New York. 277-312.

Young, E.D. and Voigt, H.F. (1982) Response properties of type II and type III units in dorsal cochlear nucleus. Hear. Res. 6, 153 169. 